Supporting Information for

\title{
Versatile labeling and detection of endogenous proteins using tag-assisted split enzyme complementation
}

Suraj Makhija, David Brown, Rachel M. Rudlaff, Julia K. Doh, Struan Bourke, Yina Wang, Shuqin Zhou, Rasmi Cheloor-Kovilakam, Bo Huang 


\section{SUPPLEMENTARY METHODS}

\section{Cloning}

We generated 'part' vectors, 'expression' vectors, and 'landing pad' vectors following the Mammalian Toolkit (MTK) approach ${ }^{18}$. The $10 \mu \mathrm{L}$ reactions to generate part vectors consisted of $40 \mathrm{fmol}$ insert DNA clean of Bsal and BsmBI restriction sites, 20 fmol MTK part vector backbone, 10x T4 Ligase Buffer (NEB B0202S), Esp31 (NEB R0734S/L), and T7 DNA Ligase $(\mathrm{M} 0318 \mathrm{~S} / \mathrm{L})$. The reactions were cycled between digestion at $37^{\circ} \mathrm{C}$ for 2 minutes and ligation at $25^{\circ} \mathrm{C}$ for 5 minutes. From the resulting reaction mixture, $1 \mu \mathrm{L}$ was transformed into Machl $E$. coli (QB3 Macrolab) and colonies lacking GFP expression were selected for amplification and sequencing verification.

To streamline cloning of the expression vectors, transcriptional unit specific CDS backbones were generated by adding the requisite connector sequences, a PGK promoter, a BGH terminator and poly $(\mathrm{A})$ to the original MTK assembly backbone, also known as pYTK095 (Addgene \#65202). With these backbones, we improved workflows by reducing the number of inserts needed to generate new assemblies. Expression vectors were generated in $10 \mu \mathrm{L}$ reactions containing $20 \mathrm{fmol}$ CDS backbone, $40 \mathrm{fmol}$ of each part insert, 10x T4 Ligase Buffer, Bsal-HF v2.0 (NEB R3733S/L), and T7 DNA Ligase with the same cycling conditions as the part vectors.

Landing pad vectors were generated similarly to part vectors in $10 \mu \mathrm{L}$ reactions with 20 fmol MTK landing pad entry backbone (Addgene \#123932), $40 \mathrm{fmol}$ of each expression vector plasmids, 10x T4 Ligase Buffer (NEB B0202S), Esp31 (NEB R0734S/L), and T7 DNA Ligase (M0318S/L) with the same cycling conditions as the part vectors.

TA-splitHalo BFP plasmids were made in $10 \mu \mathrm{L}$ reactions comprising 20 fmol Kanamycin ColE1 digested backbone, $40 \mathrm{fmol}$ TA-splitHalo fusion expression vectors, $40 \mathrm{fmol}$ PGK-mTagBFP2 expression vector, 10x T4 Ligase Buffer (NEB B0202S), Esp31 (NEB R0734S/L), and T7 DNA Ligase (NEB M0318S/L) with the same cycling conditions as the part vectors.

\section{Transfection of HeLa cells in 8-well chamber flasks for TA-splitHalo architecture benchmarking}

For Figure 1 transfections, HeLa cells were seeded in an 8-well chamber flask at 20k cells per well in $225 \mu \mathrm{L}$ DMEM +1\% Penicillin/Streptomycin (P/S) 10\% Fetal Bovine Serum (FBS). $160 \mathrm{ng}$ of each TA-splitHalo architecture plasmid was cotransfected with $80 \mathrm{ng}$ of mCherry bait plasmid with $0.7 \mu \mathrm{L}$ FuGENE HD. This corresponded to a 1:1 molar ratio. After an overnight incubation, samples were stained with $10 \mathrm{nM}$ JF646 in $100 \mu \mathrm{L}$ Phenol Red-free DMEM +1\%

Penicillin/Streptomycin $+10 \%$ Fetal Bovine Serum. Flow cytometry was performed the day after overnight staining.

\section{Seeding and transfection of HEK293T KIs for TA-splitHalo sorting}

In all experiments 6 -well chamber flasks were seeded with $300 \mathrm{k}$ pre-sorted $\mathrm{KI}$ cells and controls were seeded in $2 \mathrm{~mL}$ of DMEM $+1 \%$ P/S $10 \%$ FBS.

For Figure 4G, $180 \mathrm{fmol}$ of AS04 BFP plasmid was transfected with $2.8 \mu \mathrm{L}$ FuGENE HD in each well containing control HEK2993Ts and pre-sorted LMNA KI cells.

For Figure 6D in AS04 cells, 600 fmol of GFP1-10 plasmid was transfected with $9.3 \mu \mathrm{L}$ FuGENE $\mathrm{HD}$ in each well containing control AS04 cells and pre-sorted LMNA KI AS04 cells. 
For Figure 6G-H, $600 \mathrm{fmol}$ of GFP1-10 and 180fmol of AS04 BFP plasmid were co-transfected with $9.3 \mu \mathrm{L}$ FuGENE HD in each well containing control AS04 cells and pre-sorted LMNA KI AS04 cells.

In all cases, cells were stained in 10nM JF646 in $1 \mathrm{~mL}$ of Phenol Red-free DMEM $+1 \%$ P/S 10\% FBS after an overnight transfection. Cells were FACS sorted the day after staining.

\section{Seeding and transfecting HEK293Ts for TA-splitHalo imaging}

In all experiments, 8-well chamber flasks were pre-treated with poly-L-lysine seeding.

For AS04 BFP imaging in Figure 4H, 20k HEK293T cells containing ALFA-LMNA SpyT-LMNA $\mathrm{KIs}$ were seeded in each well. After incubation overnight, $15 \mathrm{fmol}$ AS04-BFP was transfected with $0.7 \mu \mathrm{L}$ FuGENE HD.

For AS04 cell imaging in Figure 6E, 20k sorted AS04 cells containing ALFA-LMNA SpyT-LMNA $\mathrm{KIs}$ were seeded in each well. After incubation overnight, we performed $50 \mathrm{fmol}$ transfections of GFP1-10 and GFP1-10-nHalo with 0.7 $\mu$ L FuGENE HD in different wells.

For AS04 cell imaging in Figure 6H, 20k sorted HEK293T cells containing ALFA-LMNA SpyTLMNA KIs were seeded in each well. After incubation overnight, we performed $15 \mathrm{fmol}$ GS07 BFP, $15 \mathrm{fmol}$ AS04 BFP, and 50fmol GFP1-10 + $15 \mathrm{fmol}$ AS04 BFP transfections with $0.7 \mu \mathrm{L}$ FuGENE HD in different wells.

For competing mCherry interaction experiments in Figure S7, 20k sorted HEK293T cells containing MAX-ALFA MYC-SpyT KIs were seeded in each well. After incubation overnight, we performed $25 \mathrm{fmol}$ transfections of SpyT-mCherry and ALFA-SpyT-mCherry with $0.7 \mu \mathrm{L}$ FuGENE HD in different wells.

After each of these transfections, Cells were stained with 10 nM JF646 after an overnight incubation in $100 \mu \mathrm{L}$ Phenol Red-free DMEM +1\% Penicillin/Streptomycin 10\% Fetal Bovine Serum and imaged the subsequent day.

\section{Cell line genotyping}

Genomic DNA was prepared from 1 million cells using the Monarch Genomic DNA Purification Kit (NEB, \#T3010G). Diagnostic PCR was then carried out followed by gel extraction (NucleoSpin) and Sanger Sequencing (Quintara Biosciences).

\section{qPCR validation of knock-in cell lines}

Total RNA was extracted from 1 million cells using the Monarch Total RNA Miniprep Kit (NEB, \#T2010S). We prepared cDNA from $1 \mu \mathrm{g}$ of extracted RNA using LunaScript ${ }^{\circledR}$ RT SuperMix Kit (NEB, \#E3010). No Template and No Reverse Transcriptase controls (NTC and NRT) were performed in parallel to cDNA preparations. We set up qPCR plates using $0.5 \mu \mathrm{l}$ of each $20 \mu \mathrm{l}$ cDNA sample, $10 \mu \mathrm{l}$ 2x Maxima SYBR Green qPCR Master Mix (Thermo Scientific K0221), and optimized primer pairs corresponding to SpyT-specific, GFP ${ }_{11}$-specific, and ALFA-specific LMNA KIs. We also ran a primer set specific to the wild-type LMNA gene for a positive control and reference marker.

For standard curves, we cloned plasmids containing sequences corresponding to all edited and unedited versions of the LMNA gene. RT-qPCR was performed on QuantStudio ${ }^{\text {TM }} 5$ Real-Time PCR System. These primer sequences and a schematic for our qPCR experiments are shown in Figure S10. 
SUPPLEMENTARY FIGURES

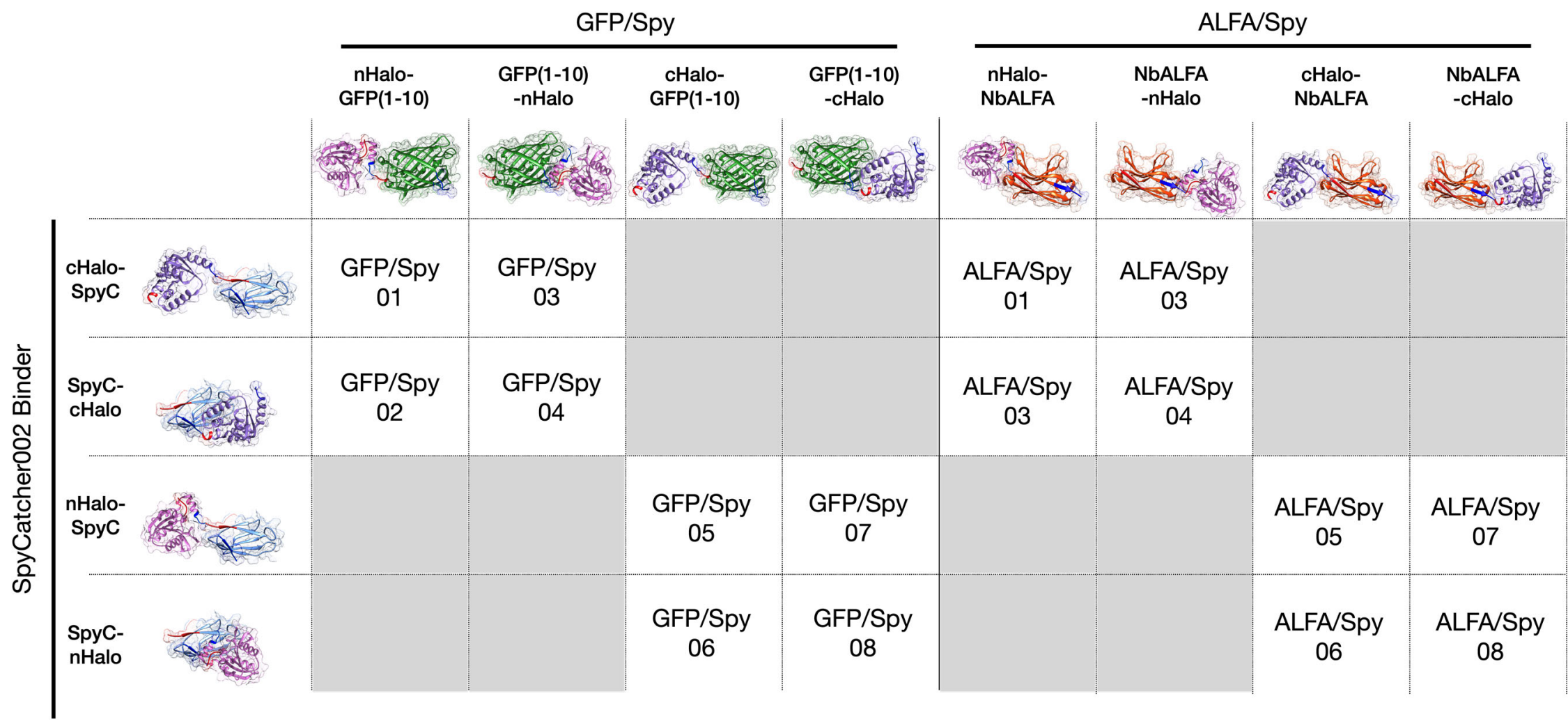

Figure S1: The relationship between TA-splitHalo fusions and architecture nomenclature. Numberings for each architecture define where the $\mathrm{nHalo}$ and cHalo components are positioned relative to each of the binders. Structural representations of TA-splitHalo fusions were made using UCSF Chimera. Components include nHalo (magenta PDB: 5UXZ), cHalo (purple PDB: 5UXZ), GFP(1-10) (green PDB 2B3P), NbALFA (PDB: orange 6I2G), and SpyCatcher002 (cyan PDB: 4MLI). N- and C-termini of all proteins are shown in red and dark blue respectively. 


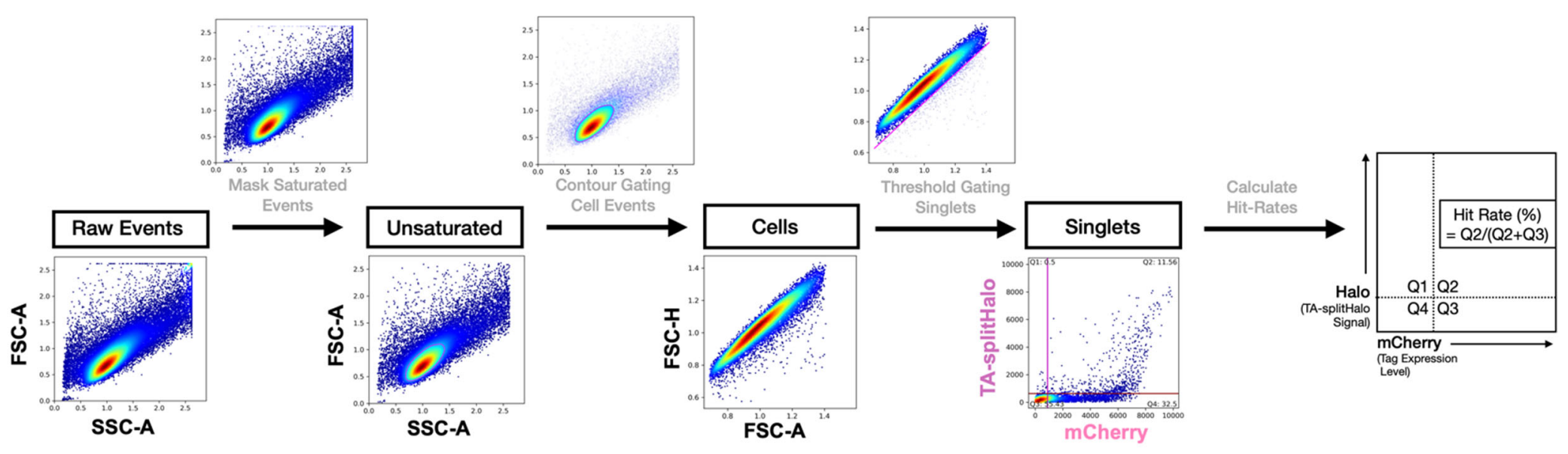

\begin{tabular}{|c|c|c|c|c|c|c|c|c|c|c|c|c|c|c|c|c|c|c|c|c|c|c|c|}
\hline \multicolumn{3}{|c|}{ GS01 } & \multicolumn{3}{|c|}{ GS02 } & \multicolumn{3}{|c|}{ GS03 } & \multicolumn{3}{|c|}{ GS04 } & \multicolumn{3}{|c|}{ GS05 } & \multicolumn{3}{|c|}{ GS06 } & \multicolumn{3}{|c|}{ GS07 } & \multicolumn{3}{|c|}{ GS08 } \\
\hline t-value & p-value & $p<.05$ & $\mathrm{t}$-value & \begin{tabular}{|l|l|}
$p$-value \\
\end{tabular} & $p<.05$ & t-value & p-value & $p<.05$ & $\mathrm{t}$-value & \begin{tabular}{|l|} 
p-value \\
\end{tabular} & $p<.05$ & $t$-value & p-value & $p<.05$ & t-value & \begin{tabular}{|l|l|}
$p$-value \\
\end{tabular} & $p<.05$ & t-value & p-value & $p<.05$ & t-value & p-value & $p<.05$ \\
\hline-7.65 & $1.27 \mathrm{E}-02$ & TRUE & -21.01 & 6.14E-04 & TRUE & -7.41 & $1.69 \mathrm{E}-02$ & TRUE & -6.68 & $8.43 \mathrm{E}-03$ & TRUE & -6.92 & $9.17 \mathrm{E}-03$ & TRUE & -5.27 & 2.28E-02 & TRUE & -6.16 & 2.44E-02 & TRUE & -3.46 & $2.66 \mathrm{E}-02$ & TRUE \\
\hline \multicolumn{3}{|c|}{ AS01 } & \multicolumn{3}{|c|}{$\underline{\mathrm{ASO}} 2$} & \multicolumn{3}{|c|}{$\underline{\mathrm{ASO} 3}$} & \multicolumn{3}{|c|}{ AS04 } & \multicolumn{3}{|c|}{ AS05 } & \multicolumn{3}{|c|}{ AS06 } & \multicolumn{3}{|c|}{ AS07 } & \multicolumn{3}{|c|}{ AS08 } \\
\hline t-value & p-value & $p<.05$ & $t$-value & p-value & $p<.05$ & $t$-value & p-value & $p<.05$ & $\mathrm{t}$-value & p-value & $p<.05$ & $t$-value & p-value & $p<.05$ & $\mathrm{t}$-value & p-value & $p<.05$ & t-value & p-value & $p<.05$ & $\mathrm{t}$-value & p-value & $p<.05$ \\
\hline-2.95 & $6.2 \mathrm{E}-02$ & FALSE & -9.83 & 6.79E-04 & TRUE & $\mid-19.63$ & $5.78 \mathrm{E}-05$ & TRUE & -13.33 & $3.28 \mathrm{E}-03$ & TRUE & -7.62 & $1.4 \mathrm{E}-02$ & TRUE & -8.43 & $5.4 \mathrm{E}-03$ & TRUE & -6.06 & $4.9 \mathrm{E}-03$ & TRUE & -4.98 & $3.80 \mathrm{E}-02$ & TRUE \\
\hline
\end{tabular}

Figure S2: Workflow diagram showing flow cytometry analysis for Figure 2 with the altFACS python package. Raw events from .fcs files are processed by eliminating saturating events, gating for singlet cell events, and deriving hit rates by assessing the fraction of TA-splitHalo+ mCherry+ cells in the total mCherry+ population. The table shows the results of Welch's unequal variance t-test for the data in Figure 2. 


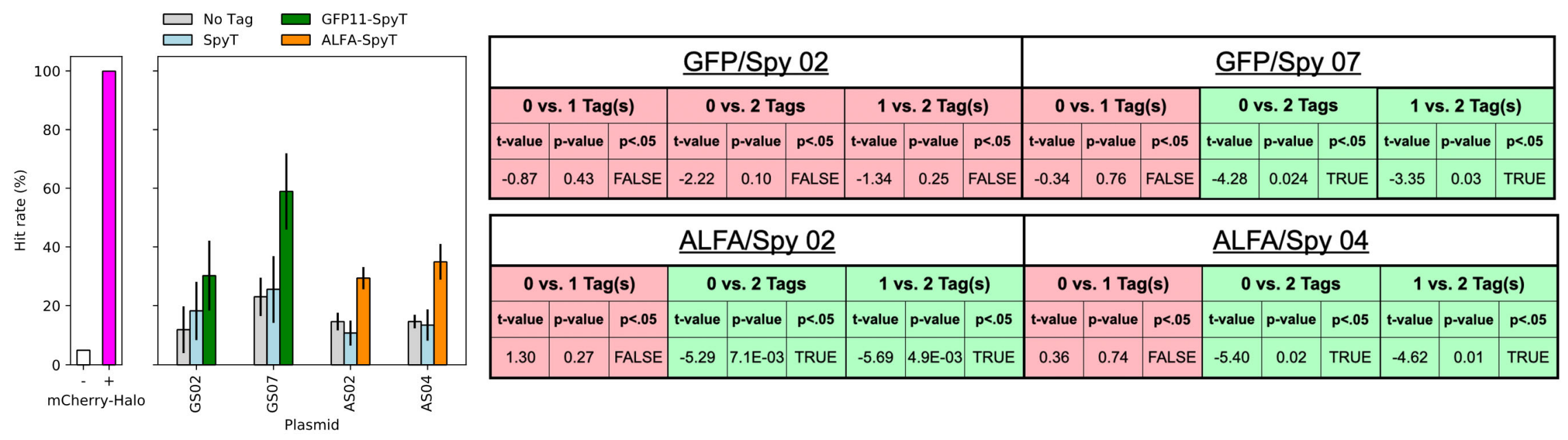

Figure S3: Bar plot investigating the TA-splitHalo tag-independent background. Compared to mCherry-HaloTag conditions without (-) and with (+) JF646 (left panel), selected architectures (right panel) have similar quantities of TA-splitHalo background when mCherry is untagged (grey) or tagged with only SpyT (blue). This implies that background is not driven by any single tag but rather by spontaneous complementation of TA-splitHalo fusions. For bars in the righthand panel, $n=3$ and errors represent standard deviation. The table shows the results of Welch's unequal variance t-test. With two tags (green and orange), we see statistically higher TAsplitHalo complementation and signal demonstrating specificity. 

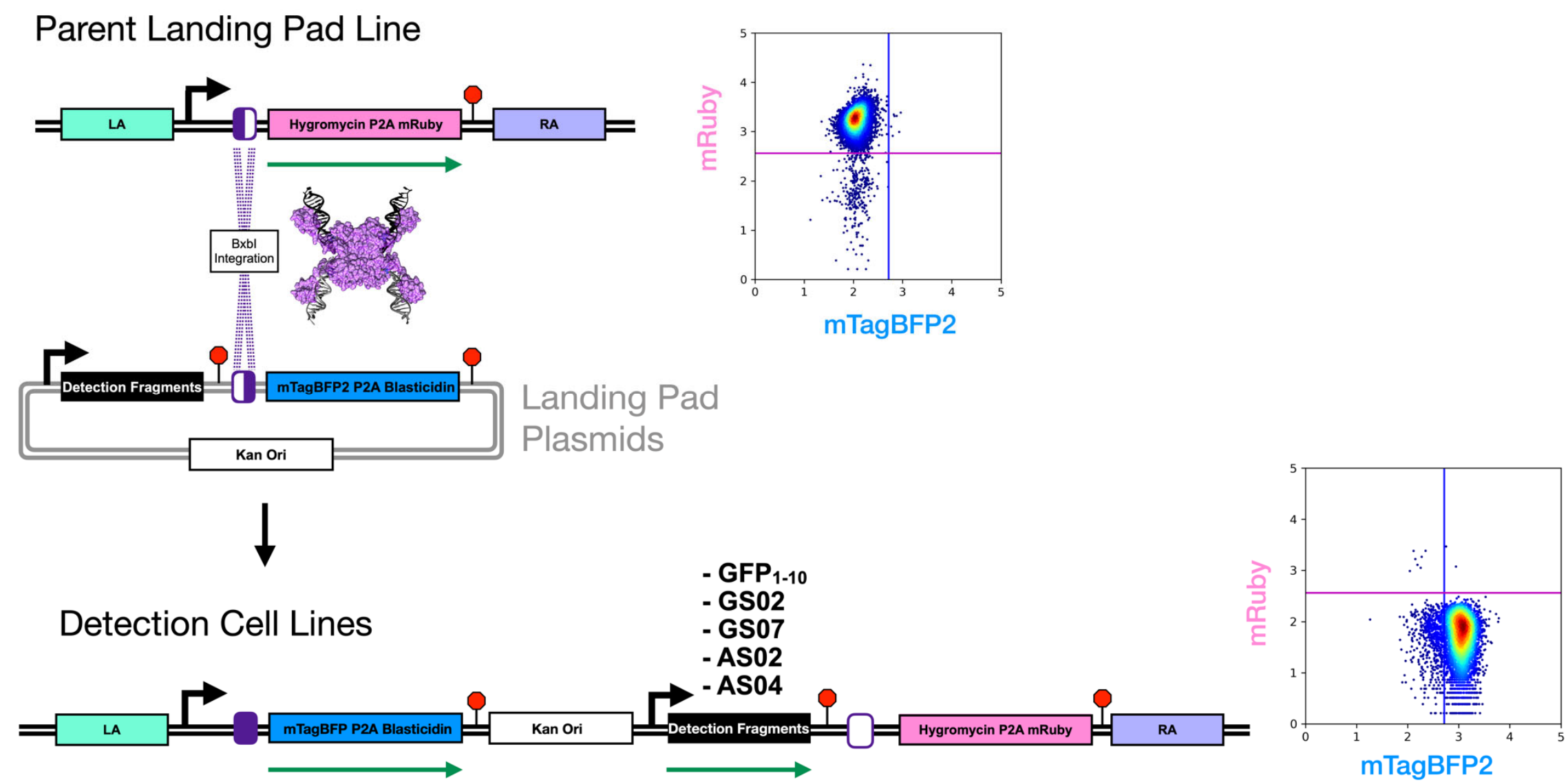

Figure S4: Isogenic cell lines were made from a parent cell line containing a Hygromycin-P2A-mRuby (pink) cassette at the AAVS1 safe harbor locus (top right). Flow cytometry confirms these cells as mRuby+ mTagBFP- (top right). Through Bxbl integration, landing pad plasmids were integrated at this site (center). Upon successful integration, the selection/reporter cassette swaps to mTagBFP2P2A-Blasticidin (blue) and detection component cargo will be expressed from a single genomic copy (black) (bottom left). Flow cytometry data shows an example of integrant cells that are mRuby- mTagBFP+ (bottom right). The left and right arms of the AAVS1 locus are shown in sea green and lavender. A structural representation of the serine integrase strand exchange between attB and attP sites (purple /is shown in magenta (PDB: 1ZR4). Promoters are shown with black arrows, ORFs that are transcribed and translated are shown with green arrows, and terminators are depicted as red stop signs. 

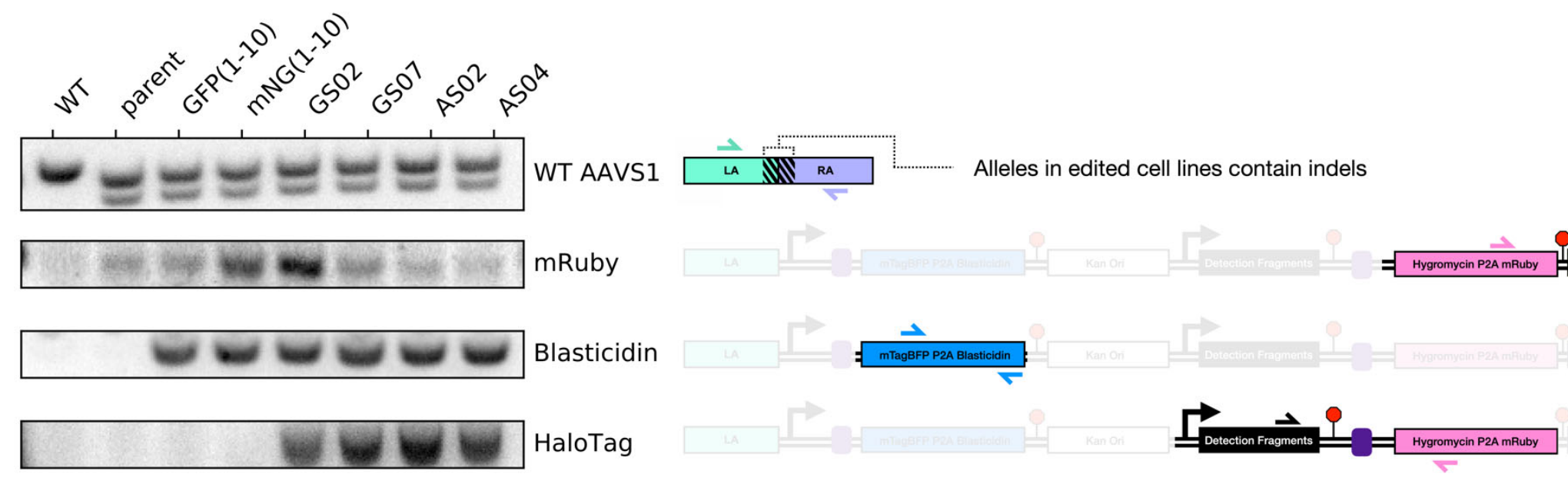

\begin{tabular}{|c|c|c|c|c|}
\hline Primer Set & Fwd Primer & Fwd Primer Sequence & Rev Primer & Rev Primer Sequence \\
\hline WT AAvs1 & Aavs1_Fwd & CTGGACAACCCCAAAGTACC & AAvs1_Rev & AGATGGCTCCAGGAAATGG \\
\hline mRuby & mRuby_Fwd & TCTTGTGCCCAGGAGAGC & mRuby_Rev & CCCAATATCAGGAGACTAGGAAGG \\
\hline Blasticidin & Blast_Fwd & CCCTCATTGAAAGAGCAACG & Blast_Rev & CCTTCACTATGGCTTTGATCC \\
\hline HaloTag & Halo_Fwd & GTCGAGATGGACCATTACCG & Halo_Rev & GGGAGATGCAATAGGTCAGG \\
\hline
\end{tabular}

Figure S5: Genotyping single integrant cell lines (left). Genomic DNA PCRs were performed to amplify the AAVS1 locus present in all cell lines $\left(1^{\text {st }}\right.$ panel), mRuby present in each landing pad parent derived lines $\left(2^{\text {nd }}\right.$ panel), blasticidin integrant present in each integrant line ( $3^{\text {rd }}$ panel), and Halo-specific integrants present in TA-splitHalo detection cell lines $\left(4^{\text {th }}\right.$ panel). The mNG(1-10) cell line made in parallel was not used in this study. Schematics defining locations of primers (black arrows) for each PCR are shown (right). 


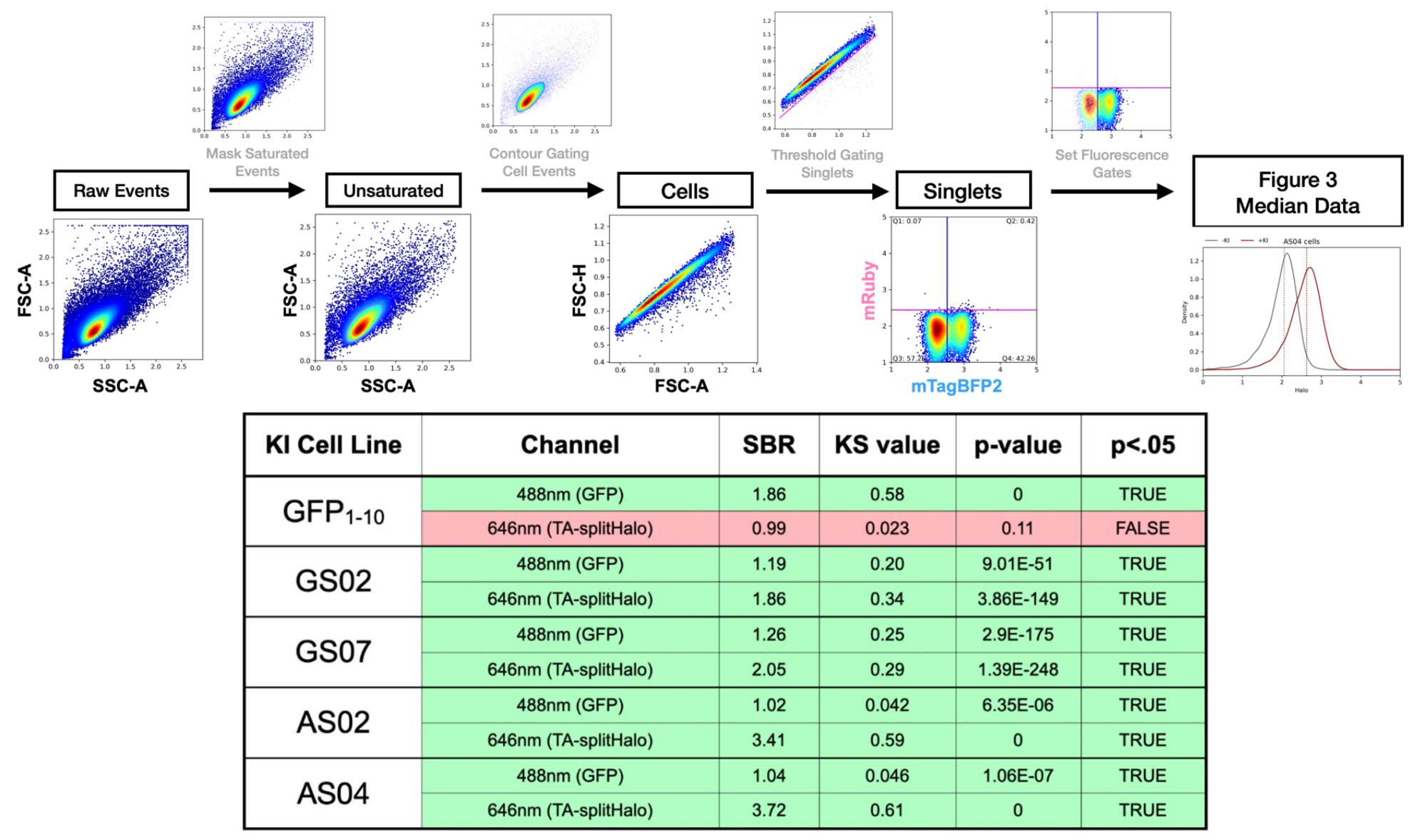

Figure S6: Workflow diagram depicting flow cytometry analysis for Figure 3 with the altFACS python package. Raw events from .fcs files are processed by eliminating saturating events, gating for singlet cell events, and setting a gate to only include mRuby-BFP+ single-copy integrant cells. From this population, we derived median GFP and TA-splitHalo signal from 10k cell events. We employed a two sample Kolmogorov-Smirnov test to assess the significance of differences between the non-normally distributed populations in our data. The null hypothesis was that the signal from the knock-in population $(+\mathrm{KI})$ is lower than the signal from the population with no knock-in (-KI). As an example, for the GFP ${ }_{1-10}$ stable cell line, we can reject that null hypothesis with high confidence in the 488nm channel. For each cell line, we also calculated the signal to background ratio (SBR) in $488 \mathrm{~nm}$ and $646 \mathrm{~nm}$ channels, by dividing the median fluorescence intensity of the $+\mathrm{KI}$ cells by the median of $-\mathrm{KI}$ cells in the same cell line. 


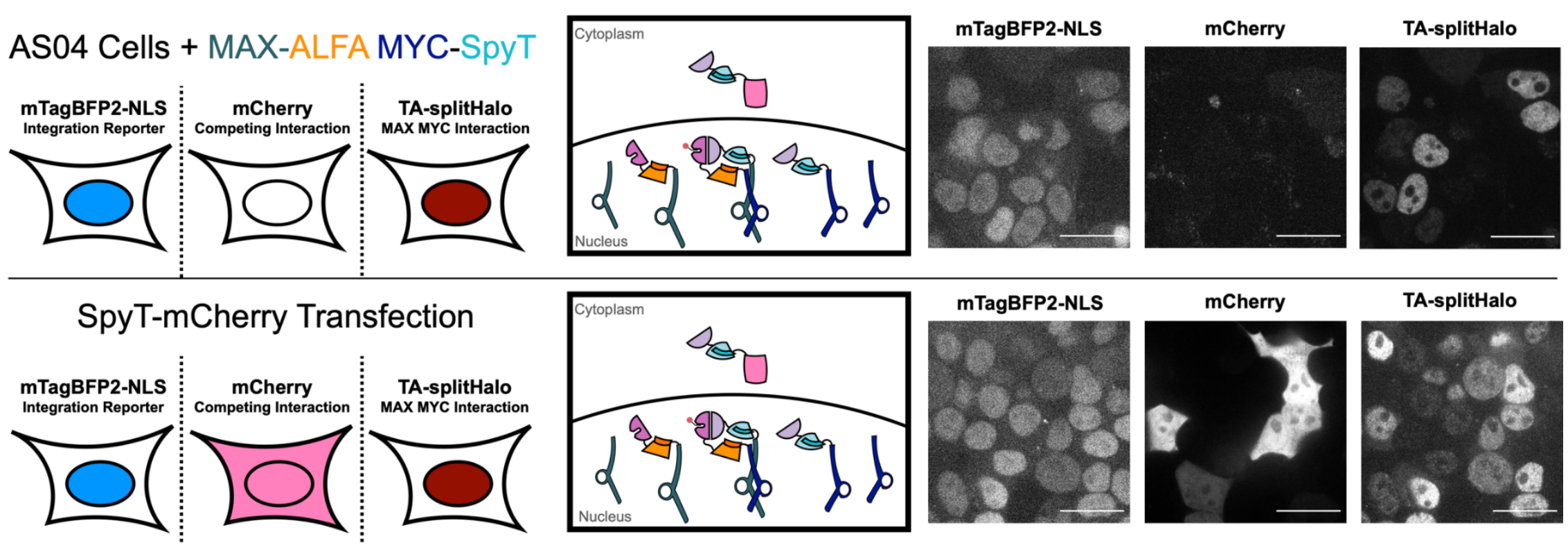

Figure S7: TA-splitHalo does not report artificial PPIs with tagged non-interactors. In AS04 cells with MAX-ALFA and MYC-SpyT KIs, TA-splitHalo signal is localized to the nucleus where MAX and MYC transcription factors interact (top panel). When SpyT-mCherry is transfected, we see that the mCherry signal is cytoplasmic at a range of expression levels and TA-splitHalo does not cause artificial nuclear enrichment of mCherry even in cells with low mCherry expression, i.e. without saturation of potential binders (bottom panel). 


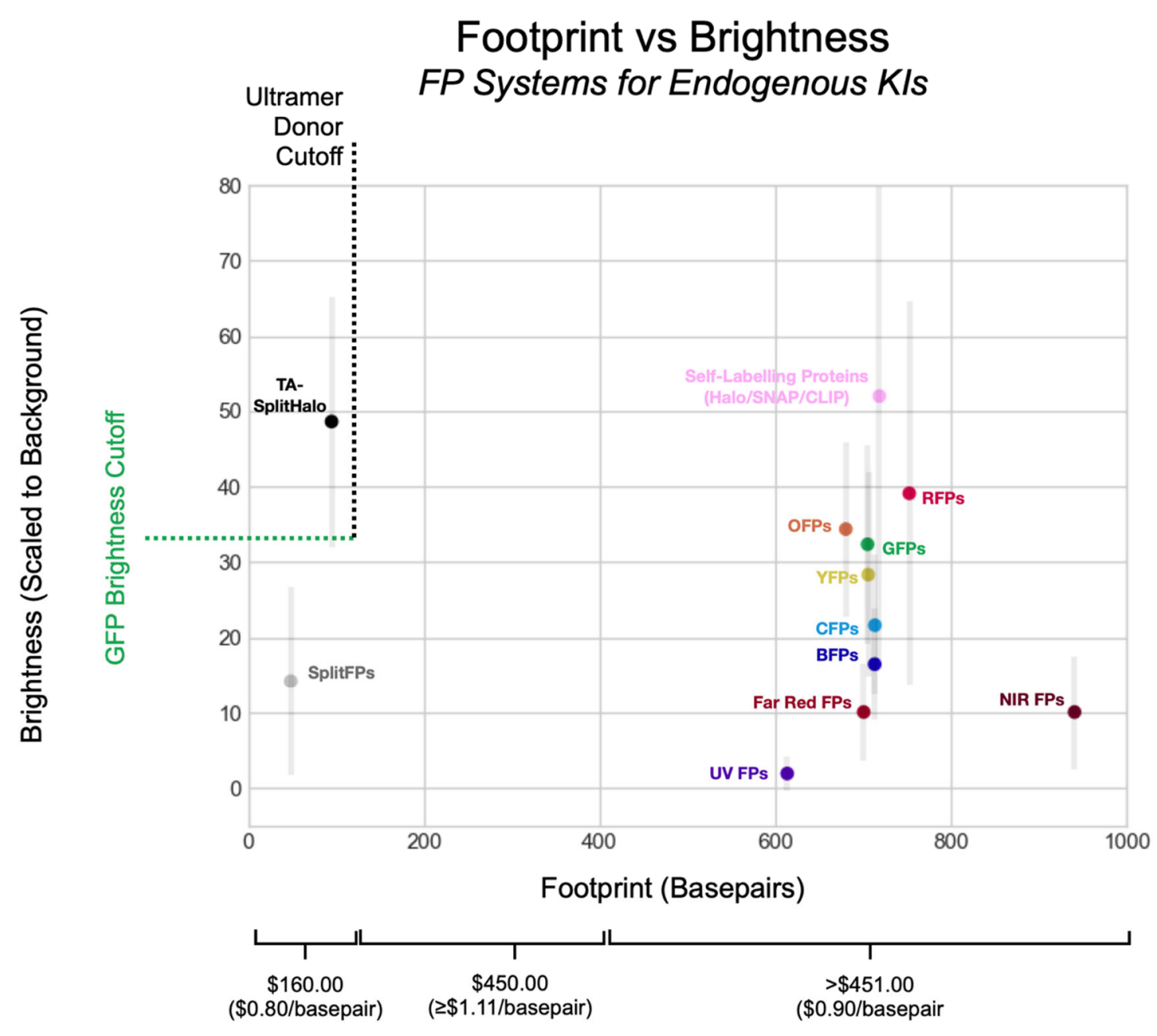

Figure S8: Scatterplot showing average brightness of fluorophores with respect to genomic footprint in base pairs and cost of producing ssDNA at given lengths. As shown, TA-splitHalo systems is the only platform that retains the cutoff for using ultramer size ssDNA donors while improving on the brightness of full-length fluorescent proteins on average. 


\begin{tabular}{|l|l|l|}
\hline Primer Name & Primer Sequence & Notes \\
\hline ML557 & $\begin{array}{l}\text { TAATACGACTCACTATAG } \\
\text { ML558 }\end{array}$ & Forward amplification primer. \\
\hline $\begin{array}{l}\text { LMNA-Specific } \\
\text { Oligo }\end{array}$ & $\begin{array}{l}\text { TAATACGACTCACTATAGCCATGGAGACCCCGTC } \\
\text { CCAGGTTTAAGAGCTATGCTGGAA }\end{array}$ & $\begin{array}{l}\text { Contains T7 transcription site LMNA- } \\
\text { specific protospacer sequence. }\end{array}$ \\
\hline ML611 & $\begin{array}{l}\text { AAAAAAAGCACCGACTCGGTGCCACTTTTT } \\
\text { CAAGTTGATAACGGACTAGCCTTATTAAACTTGC }\end{array}$ & Contains constant gRNA stem-loop. \\
\hline
\end{tabular}

\section{ML557}

TAATACGACTCACTATAG $\rightarrow$

TAATACGACTCACTATAGCCATGGAGACCCCGTCCCAGGTTTAAGAGCTATGCTGGAA $\rightarrow$

LMNA-Specific Oligo

$\leftarrow$ CAAATTCTCGATACGACCTTTGTCGT

CACGAAAAAAA

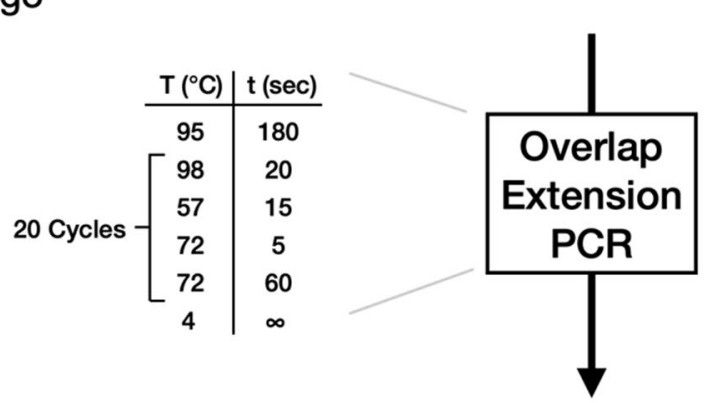

ML558

TAATACGACTCACTATAGCCATGGAGACCCCGTCCCAGGTTTAAGAGCTATGCTGGAAACAGCATAGCAAGTTTAAATAAGGCTAGTCCGTTATCAACTTGAAAAAGTGGCACCGAGTCGGTGCTTTTTTT ATTATGCTGAGTGATATCGGTACCTCTGGGGCAGGGTCCAAATTCTCGATACGACCTTTGTCGTATCGTTCAAATTTATTCCGATCAGGCAATAGTTGAACTTTTTCACCGTGGCTCAGCCACGAAAAAAA
T7
Promoter
Gene Specific
Sequence

\section{Constant Stem Loop Region}

Figure S9: Methods detailing gRNA IVT template synthesis. Primer names, sequences, and contributions are shown (top) as is the PCR amplification scheme and final IVT template for LMNA gRNA generation containing the T7 promoter (blue), LMNA-specific PAM sequence (green), and constant stem-loop region (black) (bottom). 


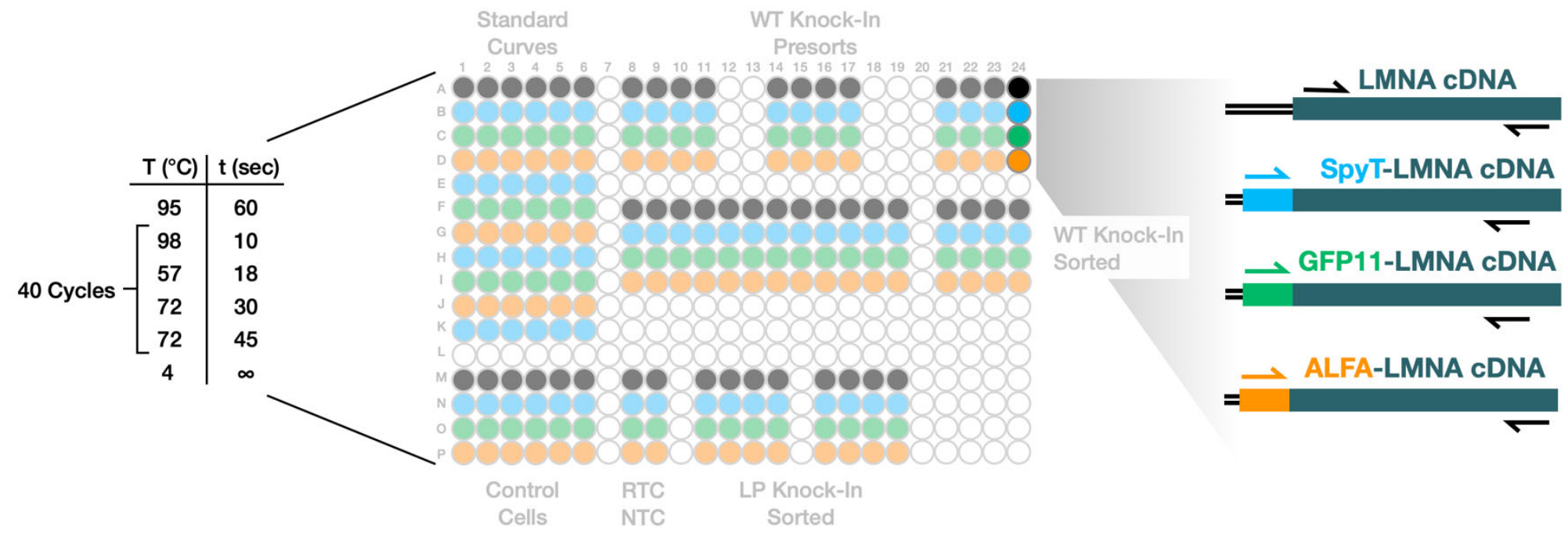

\begin{tabular}{|c|c|r|r|r|r|}
\hline Primer Set & Fwd Primer & $\underline{\text { Fwd Primer Sequence }}$ & $\underline{\text { Rev Primer }}$ & $\underline{\text { Rev Primer Sequence }}$ & Notes \\
\hline Internal & LMNA_Fwd & CAGCTCCACTCCGCTGTC & LMNA_Rev & CGCTCCTTGGCTACTGAGTC & $\begin{array}{l}\text { Amplifies all Lamin AVC } \\
\text { mRNA }\end{array}$ \\
\hline SpyT & SpyT-Fwd & CGCCTACAAGCGTTACAAGG & SpyT-Rev & CGCTCCTTGGCTACTGAGTC & Amplifies all SpyT-LMNA KIs \\
\hline GFP11 & GFP11-Fwd & CGTGACCACATGGTCCTTC & GFP11-Rev & CTGACCACCTCTTCAGACTCG & $\begin{array}{l}\text { Amplifies all GFP11-LMNA } \\
\text { Kls }\end{array}$ \\
\hline ALFA & ALFA-Fwd & GCCGACGATTGACTGAGC & ALFA-Rev & CTGACCACCTCTTCAGACTCG & Amplifies all ALFA-LMNA KIs \\
\hline
\end{tabular}

Figure S10: qPCR layout is shown (top). Control and knock-in samples from all experiments are run using four internal (black), SpyT (blue), GFP11 (green), and ALFA (orange) primer sets on the same 384-well PCR plate. The internal primer set provides a readout of LMNA cDNA abundance which the KI specific sets can be compared to. Standard curves were produced using a 1:10 dilution series of plasmids containing the predicted amplicon. 


\section{SUPPLEMENTARY TABLES}

Table S1: Gene-specific primers for gRNA IVT template PCR for heterozygous PPI targets.

Primers were used in the same manner as the LMNA primer in Figure S9 in IVT PCR reactions.

\begin{tabular}{|c|c|c|}
\hline Primer Name & Primer Sequence & Notes \\
\hline $\begin{array}{l}\text { TUBA1B-Specific } \\
\text { Oligo }\end{array}$ & $\begin{array}{l}\text { TAATACGACTCACTATAGGATGCACTCACGCTGCGG } \\
\text { GAGTTTAAGAGCTATGCTGGAA }\end{array}$ & $\begin{array}{l}\text { Contains T7 transcription site and } \\
\text { TUBA1B-specific protospacer sequence. }\end{array}$ \\
\hline $\begin{array}{l}\text { TUBB4B-Specific } \\
\text { Oligo }\end{array}$ & $\begin{array}{l}\text { TAATACGACTCACTATAGCGCCGCCGCCGCCATCAT } \\
\text { GAGTTTAAGAGCTATGCTGGAA }\end{array}$ & $\begin{array}{l}\text { Contains T7 transcription site and } \\
\text { TUBB4B-specific protospacer sequence. }\end{array}$ \\
\hline $\begin{array}{l}\text { RAD21-Specific } \\
\text { Oligo }\end{array}$ & $\begin{array}{l}\text { TAATACGACTCACTATAGCCAAGGTTCCATATTATATA } \\
\text { GTTTAAGAGCTATGCTGGAA }\end{array}$ & $\begin{array}{l}\text { Contains T7 transcription site and RAD21- } \\
\text { specific protospacer sequence. }\end{array}$ \\
\hline $\begin{array}{l}\text { SMC1A-Specific } \\
\text { Oligo }\end{array}$ & $\begin{array}{l}\text { TAATACGACTCACTATAGCCGGCGCCGCGGCCGTCA } \\
\text { TGGTITAAGAGCTATGCTGGAA }\end{array}$ & $\begin{array}{l}\text { Contains T7 transcription site and } \\
\text { SMC1A-specific protospacer sequence. }\end{array}$ \\
\hline $\begin{array}{l}\text { PSME4-Specific } \\
\text { Oligo }\end{array}$ & $\begin{array}{l}\text { TAATACGACTCACTATAGCCTTTCTATGCATAATAGCA } \\
\text { GTTTAAGAGCTATGCTGGAA }\end{array}$ & $\begin{array}{l}\text { Contains T7 transcription site and } \\
\text { PSME4-specific protospacer sequence. }\end{array}$ \\
\hline $\begin{array}{l}\text { PSMB2-Specific } \\
\text { Oligo }\end{array}$ & $\begin{array}{l}\text { TAATACGACTCACTATAGGATGTTAGGAGCCCTGTTT } \\
\text { GGTTTAAGAGCTATGCTGGAA }\end{array}$ & $\begin{array}{l}\text { Contains T7 transcription site and } \\
\text { PSMB2-specific protospacer sequence. }\end{array}$ \\
\hline $\begin{array}{l}\text { PSMA3-Specific } \\
\text { Oligo }\end{array}$ & $\begin{array}{l}\text { TAATACGACTCACTATAGGATGTTAGGAGCCCTGTTT } \\
\text { GGTTTAAGAGCTATGCTGGAA }\end{array}$ & $\begin{array}{l}\text { Contains T7 transcription site and } \\
\text { PSMA3-specific protospacer sequence. }\end{array}$ \\
\hline $\begin{array}{l}\text { LMNA-Specific } \\
\text { Oligo }\end{array}$ & $\begin{array}{l}\text { TAATACGACTCACTATAGCCATGGAGACCCCGTCCC } \\
\text { AGGTTTAAGAGCTATGCTGGAA }\end{array}$ & $\begin{array}{l}\text { Contains T7 transcription site and LMNA- } \\
\text { specific protospacer sequence. }\end{array}$ \\
\hline $\begin{array}{l}\text { CBX5-Specific } \\
\text { Oligo }\end{array}$ & $\begin{array}{l}\text { TAATACGACTCACTATAGGGTGGAACGGTGTGCGAC } \\
\text { ATGTTTAAGAGCTATGCTGGAA }\end{array}$ & $\begin{array}{l}\text { Contains T7 transcription site and CBX5- } \\
\text { specific protospacer sequence. }\end{array}$ \\
\hline $\begin{array}{l}\text { MAX-Specific } \\
\text { Oligo }\end{array}$ & $\begin{array}{l}\text { TAATACGACTCACTATAGGAGGCCAGCTAAGCCACT } \\
\text { CGGTTTAAGAGCTATGCTGGAA }\end{array}$ & $\begin{array}{l}\text { Contains T7 transcription site and MAX- } \\
\text { specific protospacer sequence. }\end{array}$ \\
\hline $\begin{array}{l}\text { MDX1-Specific } \\
\text { Oligo }\end{array}$ & $\begin{array}{l}\text { TAATACGACTCACTATAGTGTCTTGGTCTCTAAGAGA } \\
\text { GGTTTAAGAGCTATGCTGGAA }\end{array}$ & $\begin{array}{l}\text { Contains T7 transcription site and MDX1- } \\
\text { specific protospacer sequence. }\end{array}$ \\
\hline $\begin{array}{l}\text { MYC-Specific } \\
\text { Oligo }\end{array}$ & $\begin{array}{l}\text { TAATACGACTCACTATAGCTACGGAACTCTTGTGCGT } \\
\text { AGTITAAGAGCTATGCTGGAA }\end{array}$ & $\begin{array}{l}\text { Contains T7 transcription site and MYC- } \\
\text { specific protospacer sequence. }\end{array}$ \\
\hline
\end{tabular}


Table S2: 200 bp ultramer donor strands used for LMNA KIs. Sequences for GFP11 (green), SpyT (blue), ALFA (orange) GS linkers (black) and LMNA homology (teal) are bolded.

\begin{tabular}{|c|c|}
\hline$\underline{\text { Ultramer Name }}$ & Ultramer Sequence \\
\hline $\begin{array}{c}\text { GFP11-SpyT- } \\
\text { LMNA }\end{array}$ & $\begin{array}{l}\text { TTTCCGGGACCCCTGCCCCGCGGGCAGCGCTGCCAACCTGCCGGC } \\
\text { CATGCGTGACCACATGGTCCTTCATGAGTATGTAAATGCTGCTGGG } \\
\text { ATTACAGGTTCTGTGCCTACTATCGTGATGGTGGACGCCTACAAGC } \\
\text { GTTACAAGGGATCCGAGACCCCGTCCCAGCGGCGCGCCACCCGC } \\
\text { AGCGGGGCGCAGGCCAGCT }\end{array}$ \\
\hline ALFA-SpyT-LMNA & $\begin{array}{l}\text { CCTTTCCGGGACCCCTGCCCCGCGGGCAGCGCTGCCAACCTGCCG } \\
\text { GCCATGCCTAGCCGCCTGGAGGAAGAACTCCGCCGACGATTGACT } \\
\text { GAGCCAGGTTCTGTGCCTACTATCGTGATGGTGGACGCCTACAAG } \\
\text { CGTTACAAGGGATCCGAGACCCCGTCCCAGCGGCGCGCCACCCG } \\
\text { CAGCGGGGCGCAGGCCAGCTC }\end{array}$ \\
\hline GFP11-LMNA & $\begin{array}{l}\text { GTCCTTCGACCCGAGCCCCGCGCCCTTTCCGGGACCCCTGCCCCG } \\
\text { CGGGCAGCGCTGCCAACCTGCCGGCCATGCGTGACCACATGGTCC } \\
\text { TTCATGAGTATGTAAATGCTGCTGGGATTACAGGATCCGAGACCCC } \\
\text { GTCCCAGCGGCGCGCCACCCGCAGCGGGGCGCAGGCCAGCTCCA } \\
\text { CTCCGCTGTCGCCCACCCGC }\end{array}$ \\
\hline AFLA-LMNA & $\begin{array}{l}\text { TGTCCTTCGACCCGAGCCCCGCGCCCTTTCCGGGACCCCTGCCCC } \\
\text { GCGGGCAGCGCTGCCAACCTGCCGGCCATGCCTAGCCGCCTGGAG } \\
\text { GAAGAACTCCGCCGACGATTGACTGAGCCAGGATCCGAGACCCC } \\
\text { GTCCCAGCGGCGCGCCACCCGCAGCGGGGCGCAGGCCAGCTCCA } \\
\text { CTCCGCTGTCGCCCACCCGCAT }\end{array}$ \\
\hline SpyT-LMNA & $\begin{array}{l}\text { TCTGTCCTTCGACCCGAGCCCCGCGCCCTTTCCGGGACCCCTGCCC } \\
\text { CGCGGGCAGCGCTGCCAACCTGCCGGCCATGGTGCCTACTATCGT } \\
\text { GATGGTGGACGCCTACAAGCGTTACAAGGGATCCGAGACCCCGTC } \\
\text { CCAGCGGCGCGCCACCCGCAGCGGGGCGCAGGCCAGCTCCACTC } \\
\text { CGCTGTCGCCCACCCGCATC }\end{array}$ \\
\hline
\end{tabular}


Table S3: 200 bp ultramer donor strands used for heterodimerization KIs in AS04 cells. Sequences for SpyT (blue), ALFA (orange), GS linkers (black) and protein interactor homology (teal or navy) are bolded.

\begin{tabular}{|c|c|}
\hline Ultramer Name & Ultramer Sequence \\
\hline ALFA-TUBA1B & $\begin{array}{l}\text { TATTTTGTTCCACTTGACAGTCATCAATAGATTGGTTTAAATTGTGAT } \\
\text { ATCTTTTTTCCTCCCGCAGCCTAGCCGCCTGGAGGAAGAACTCC } \\
\text { GCCGACGATTGACTGAGCCAGGGGGCTCAGGTGGCTCCCGTGAG } \\
\text { TGCATCTCCATCCACGTTGGCCAGGCTGGTGTCCAGATTGGCAAT } \\
\text { GCCTGCTGGGAGCTCTAC }\end{array}$ \\
\hline SpyT-TUBB4B & $\begin{array}{l}\text { TGCGCGCCCGCTCTTCTGCTGCTGTTTGTCTACTTCCTCCTGCTTC } \\
\text { CCCGCCGCCGCCGCCGCCAAAATGGTGCCTACTATCGTGATGGTG } \\
\text { GACGCCTACAAGCGTTACAAGGGGGGCTCAGGTGGCTCCAGGGA } \\
\text { AATCGTGCACTTGCAGGCCGGGCAGTGCGGCAACCAAATCGGCG } \\
\text { CCAAGGTAAGTTGCCggggcg }\end{array}$ \\
\hline RAD21-ALFA & $\begin{array}{l}\text { GCAAGCTATTGAGCTGACACAGGAAGAACCGTACAGTGACATCAT } \\
\text { CGCAACACCTGGACCAAGGTTCCATATTATAGGATCCCCTAGCCG } \\
\text { CCTGGAGGAAGAACTCCGCCGACGATTGACTGAGTAAGGAGCTA } \\
\text { GAAGCATTATAGCTAGTGTTTGATTCACTAGTGCTTACAAATTGCCC } \\
\text { CCATGTGTAGGGGACACAG }\end{array}$ \\
\hline SpyT-SMC1A & $\begin{array}{l}\text { TCCCGCGCGGGCTACCTCAGTTCTCGGGCGTACGGCGCGGCCTGT } \\
\text { CCTACTGCCGCCGGCGCCGCGGCCGTCATGGTGCCTACTATCGTG } \\
\text { ATGGTGGACGCCTACAAGCGTTACAAGGGATCCGGGTTCCTGAAA } \\
\text { CTGATTGAGATTGAGAACTTTAAGTCGTACAAGGGTCGACAGATT } \\
\text { ATCGGACCATTTCAGAGGTT }\end{array}$ \\
\hline PSME4-ALFA & $\begin{array}{l}\text { GGAACATAAACAGCAATTCACTGATGACCAACTGCTTGTTCTCAC } \\
\text { CGATCTTCTTGTGTCACCATGCTATTATGCAGGATCCCCTAGCCGC } \\
\text { CTGGAGGAAGAACTCCGCCGACGATTGACTGAGTAGAAAGGTAAG } \\
\text { TCAGCAAAGTTCTGAATTTACATTGGTTTGGTGACTGAGAACTAGAT } \\
\text { ATTTATTGtttttttC }\end{array}$ \\
\hline PSMB2-SpyT & $\begin{array}{l}\text { AACCTTCAGTGTTCGAATCATTGACAAAAATGGCATCCATGACCT } \\
\text { GGATAACATTTCCTTCCCCAAACAGGGCTCCGGTTCTGTGCCTACT } \\
\text { ATCGTGATGGTGGACGCCTACAAGCGTTACAAGTAACATCATGTC } \\
\text { CTCCCTCCCACTTGCCAGGGAACtttttttgatgggctcctttattttttctactctttcA }\end{array}$ \\
\hline PSMA3-SpyT & $\begin{array}{l}\text { AAATCAATTTTAAACACCTGTTTTCTCTTAACAGGAgTCaCTGAAG } \\
\text { GAAGAAGATGAATCAGATGATGATAATATGGGTTCTGTGCCTACT } \\
\text { ATCGTGATGGTGGACGCCTACAAGCGTTACAAGTAACATTTACTCC } \\
\text { AGCATCTATTGTATTTTAAATTTCTACTCCAGTCCAATGTAACTATTT } \\
\text { AGCCCTGGATTATAC }\end{array}$ \\
\hline ALFA-CBX5 & $\begin{array}{l}\text { TTCTAACCATTTTGTCCTTTGCCTTTGCAGGGACCTGGTGGCCTTAG } \\
\text { TCTTTCAGGTGGAACGGTGTGCGACATGCCTAGCCGCCTGGAGGA } \\
\text { AGAACTCCGCCGACGATTGACTGAGCCAGGATCCGGAAAGAAAA } \\
\text { CCAAGCGGACAGCTGACAGTTCTTCTTCAGAGGATGAGGAGGAG } \\
\text { TATGTTGTGGAGAAGGTGCT }\end{array}$ \\
\hline
\end{tabular}




\begin{tabular}{|c|c|}
\hline SpyT-LMNA & $\begin{array}{l}\text { TCTGTCCTTCGACCCGAGCCCCGCGCCCTTTCCGGGACCCCTGCC } \\
\text { CCGCGGGCAGCGCTGCCAACCTGCCGGCCATGGTGCCTACTATC } \\
\text { GTGATGGTGGACGCCTACAAGCGTTACAAGGGATCCGAGACCCC } \\
\text { GTCCCAGCGGCGCGCCACCCGCAGCGGGGCGCAGGCCAGCTCC } \\
\text { ACTCCGCTGTCGCCCACCCGCATC }\end{array}$ \\
\hline MAX-ALFA & $\begin{array}{l}\text { TGGGGGCTCGGACTCCAGCTCGGAGTCTGAGCCTGAAGAGCCCC } \\
\text { AAAGCAGGAAGAAGCTCCGGATGGAGGCCAGCGGATCCCCTAGC } \\
\text { CGCCTGGAGGAAGAACTCCGCCGACGATTGACTGAGTAAGCCAC } \\
\text { TCGGGGCAGGCCAGCAATAAAAACTGTCTGTCTCCATCGTCTCATC } \\
\text { CTCCTTTCAGTTCGTTGGTAGA }\end{array}$ \\
\hline MXD1-Spy ${ }^{\top}$ & $\begin{array}{l}\text { CAGTGATGAGGGCTATTCCAGCACCAGCATCAAGAGAATAAAGC } \\
\text { TGCAGGACAGTCACAAGGCGTGTCTTGGTCTCGGTTCTGTGCCTA } \\
\text { CTATCGTGATGGTGGACGCCTACAAGCGTTACAAGTAAGAGAGTG } \\
\text { GGCACTGCGGCTGTCTCCTTGAAGGTTCTCCCTGTTGGTTCTGATT } \\
\text { AGGTAACGTATTGGACCTGC }\end{array}$ \\
\hline MYC-SpyT & $\begin{array}{l}\text { TTCTGAAGAGGACTTGTTGCGGAAACGACGAGAACAGTTGAAAC } \\
\text { ACAAACTTGAACAGCTACGGAACTCTTGTGCGGGTTCTGTGCCTA } \\
\text { CTATCGTGATGGTGGACGCCTACAAGCGTTACAAGTAAGGAAAAG } \\
\text { TAAGGAAAACGATTCCTTCTAACAGAAATGTCCTGAGCAATCACCTA } \\
\text { TGAACTTGTTTCAAATGCA }\end{array}$ \\
\hline
\end{tabular}

